\title{
Use of an objective measure of time to recovery after cardiac surgery - the STET randomised controlled trial
}

\author{
Chris A Rogers", Katie Pike, Gianni D Angelini, Barney C Reeves \\ From Clinical Trials Methodology Conference 2011 \\ Bristol, UK. 4-5 October 2011
}

\section{Objective}

The STET trial is a two-centre open RCT comparing morbidity and healthcare resource use when off-pump coronary artery bypass surgery is carried out via a left anterolateral thoracotomy incision (ThoraCAB) or via a conventional median sternotomy incision (OPCAB). It was hypothesised that post-operative recovery would be faster with ThoraCAB.

\section{Methods}

Post-operative hospital stay is a commonly used measure of recovery. However, in an open surgical trial, where the decision to discharge the patient from hospital lies with the surgeon, post operative hospital stay is susceptible to bias. For the STET trial we developed an objective measure of recovery. Recovery time was defined as the time from surgery until the patient was considered fit for discharge. Patients were classified fit (a) when the x-ray was clear (no evidence of pleural effusion requiring drainage, lung collapse/consolidation, pneumothorax); (b) there was no suspected systemic, lower respiratory tract or wound infection; (c) routine blood results and temperature were normal and (d) when physically mobile (walking $70 \mathrm{~m}$, bowels open and oxygen saturation $>95 \%$ ). These recovery criteria were monitored daily until discharge.

\section{Results}

184 patients were recruited (91 randomised to Thora$\mathrm{CAB}, 93$ to $\mathrm{OPCAB}$ ). In the OPCAB group $77 \%$ were classified fit at or before discharge versus $68 \%$ in the Thora $\mathrm{CAB}$ group. For the remainder, the recovery time was censored because discharge occurred before all the criteria were met. Insufficient mobility accounted for the majority of censored observations. The median recovery time was 6 days, IQR [4,7] in the ThoraCAB group versus 5 days, IQR $[4,7]$ in the OPCAB group (Time ratio (ThoraCAB/OPCAB) 1.03 (95\%CI [0.94, 1.14], $\mathrm{p}=0.53)$. In contrast, the median time to discharge was 5 days in the ThoraCAB group versus 6 days in the OPCAB group.

\section{Conclusion}

The anticipated faster recovery with ThoraCAB was not found and a significant proportion of patients were discharged before all the recovery criteria were met. The results from the STET trial illustrate the bias associated with clinical decision making in an open RCT. The measure of recovery time (with slightly modified criteria) is being used in other cardiac surgery trials.

Published: 13 December 2011

doi:10.1186/1745-6215-12-S1-A68

Cite this article as: Rogers et al:: Use of an objective measure of time to recovery after cardiac surgery - the STET randomised controlled trial.

Trials 2011 12(Suppl 1):A68. 